\title{
Correction to: Allogeneic hematopoietic stem cell transplantation improves long-term outcome for relapsed AML patients across all ages: results from two East German Study Group Hematology and Oncology (OSHO) trials
}

\author{
Thomas Heinicke ${ }^{1} \cdot$ Rainer $\mathrm{Krahl}^{2} \cdot$ Christoph Kahl $^{3} \cdot$ Michael Cross $^{2} \cdot$ Sebastian Scholl ${ }^{4} \cdot$ Hans-Heinrich Wolf $^{5}$. \\ Detlev Hähling ${ }^{6}$. Ute Hegenbart ${ }^{7} \cdot$ Norma Peter $^{8}$. Antje Schulze ${ }^{9}$. Axel Florschütz ${ }^{10}$ • Volker Schmidt ${ }^{9}$. \\ Kolja Reifenrath ${ }^{11}$. Niklas Zojer ${ }^{12}$. Christian Junghanss ${ }^{13}$. Herbert G. Sayer ${ }^{9}$. Georg Maschmeyer ${ }^{14}$. \\ Christian Späth ${ }^{15}$. Andreas Hochhaus ${ }^{4}$. Thomas Fischer ${ }^{1}$ - Haifa Kathrin Al-Ali ${ }^{5}$. Dietger Niederwieser2,16,17
}

Published online: 16 September 2021

๑) Springer-Verlag GmbH Germany, part of Springer Nature 2021

Correction to: Annals of Hematology https://doi.org/10.1007/s00277-021-04465-4

The first name of authors were erroneously presented (correct Volker Schmidt instead of Schmidt Volker; correct Christian Späth instead of Späth Wolfgang). In Fig. 2B, the n value of de novo AML should be 389 instead of 369. In Fig. 2D, the $n$ value of $\leq 6$ months should be 271 instead of 86 and the $n$ value of $>18$ months should be 86 instead of 271 .

The original article can be found online at https://doi.org/10.1007/ s00277-021-04565-1.

Dietger Niederwieser

dietger.niederwieser@medizin.uni-leipzig.de

1 Department of Hematology and Oncology, University of Magdeburg, Magdeburg, Germany

2 University Hospital Leipzig, 04106 Leipzig, Germany

3 Department of Hematology and Oncology, Hospital Magdeburg, Magdeburg, Germany

4 Klinik fur Innere Medizin II, Universitatsklinikum Jena, Jena, Germany

5 Department of Hematology and Oncology, University Hospital, Halle, Germany

6 Department of Hematology and Oncology, Klinikum Schwerin, Schwerin, Germany

7 Departement of Internal Medicine V, University of Heidelberg, Heidelberg, Germany

8 Medizinische Klinik, Carl-Thieme-Klinikum GmbH, Cottbus, Germany
9 Department of Hematology and Oncology, Helios Klinikum Erfurt, Erfurt, Germany

10 Klinikum Dessau, Dessau, Germany

11 Klinikum, Zittau, Germany

12 Department of Medicine I, Wilhelminen Cancer Research Institute, WilhelminenhospitalVienna, Austria

13 Hematology, Oncology, Palliative Medicine, University of Rostock, Rostock, Germany

14 Department of Hematology, Oncology and Palliative Care, Ernst Von Bergmann Hospital, Potsdam, Germany

15 Hematology and Oncology, University of Greifswald, Greifswald, Germany

16 Lithuanian University of Health Sciences, Kaunas, Lithuania

17 Aichi Medical University, Nagakute, Japan 
The original article has been corrected.
A

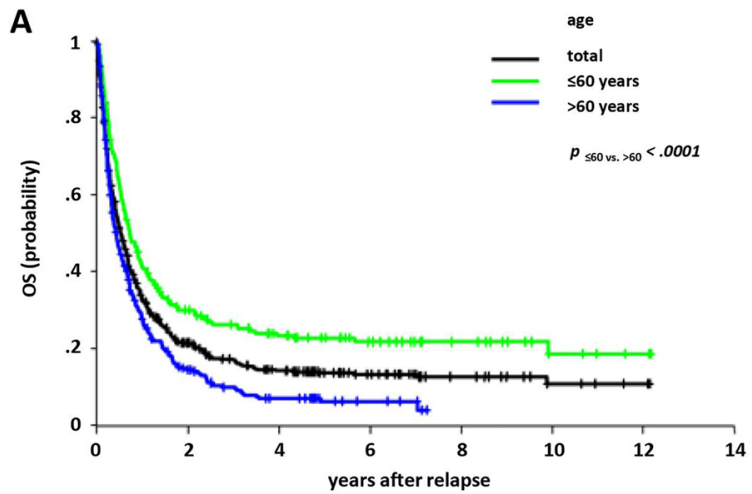

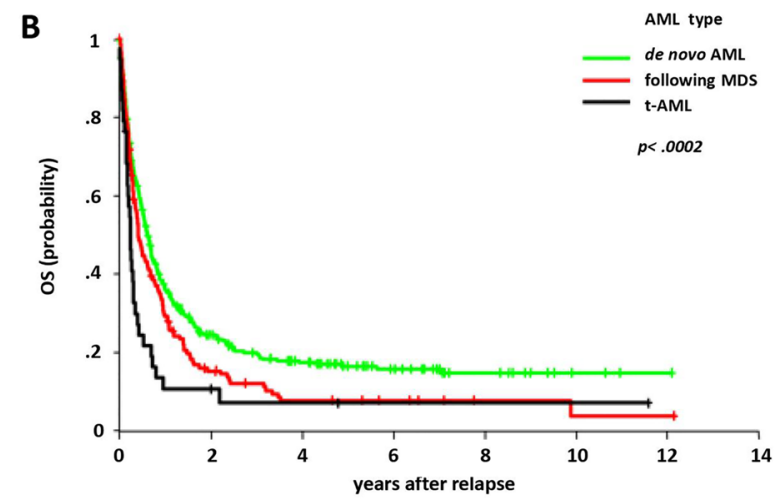

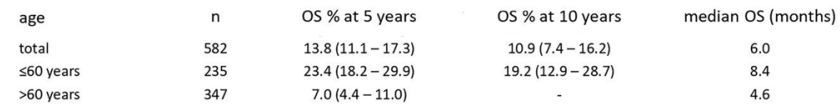

C

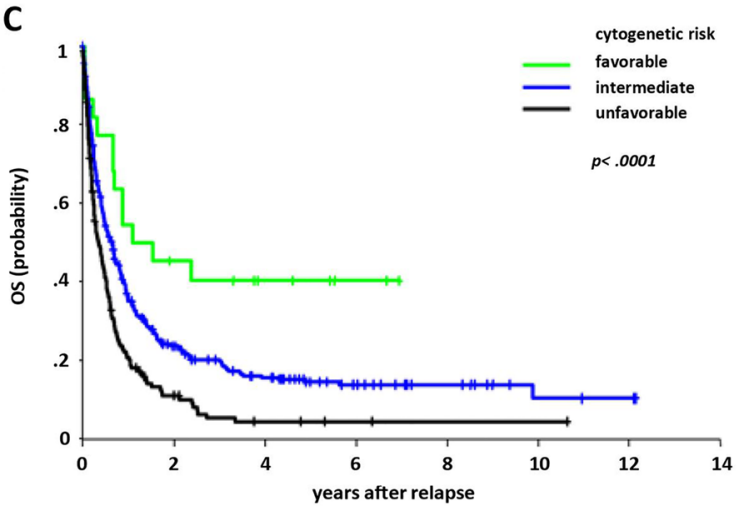

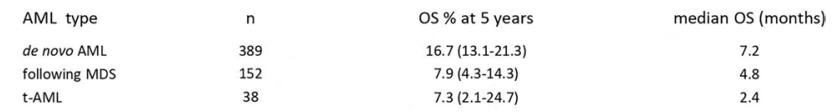

t-AML

D

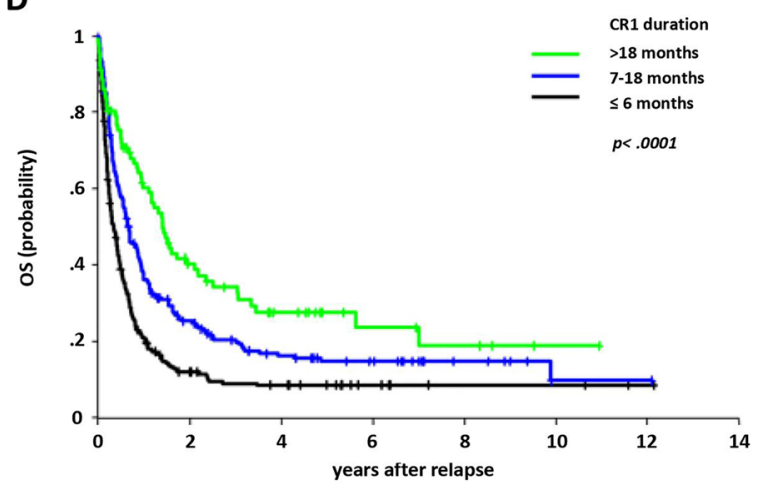

Fig. 2 (Relapsed patients). A Overall survival (OS) of patients with AML after frst relapse according to age. B Overall survival (OS) of patients with AML after frst relapse according to de novo, secondary, and therapy-related AML. C Overall survival (OS) of patients with AML after frst relapse according to favorable, intermediate, and unfavorable cytogenetics. D Overall survival (OS) of patients with AML after frst relapse according to time interval CR1 and relapse in months

Publisher's note Springer Nature remains neutral with regard to jurisdictional claims in published maps and institutional affiliations. 